\title{
Complete genome sequence of Truepera radiovictrix type strain (RQ-24 $\left.{ }^{\top}\right)$
}

\author{
Natalia Ivanova ${ }^{1}$, Christine Rohde ${ }^{2}$, Christine Munk ${ }^{1,3}$, Matt Nolan ${ }^{1}$, Susan Lucas ${ }^{1}$, Tijana \\ Glavina Del Rio ${ }^{1}$, Hope Tice ${ }^{1}$, Shweta Deshpande ${ }^{1}$, Jan-Fang Cheng ${ }^{1}$, Roxane Tapia ${ }^{1,3}$, Cliff \\ Han $^{1,3}$, Lynne Goodwin ${ }^{1,3}$, Sam Pitluck ${ }^{1}$, Konstantinos Liolios ${ }^{1}$, Konstantinos Mavromatis ${ }^{1}$, \\ Natalia Mikhailova ${ }^{1}$, Amrita Pati ${ }^{1}$, Amy Chen ${ }^{4}$, Krishna Palaniappan ${ }^{4}$, Miriam Land ${ }^{1,5}$, Loren \\ Hauser $^{1,5}$, Yun-Juan Chang ${ }^{1,5}$, Cynthia D. Jeffries ${ }^{1,5}$, Evelyne Brambilla ${ }^{2}$, Manfred Rohde ${ }^{6}$, \\ Markus Göker ${ }^{2}$, Brian J. Tindall ${ }^{2}$, Tanja Woyke', James Bristow ${ }^{1}$, Jonathan A. Eisen ${ }^{1,7}$, Victor \\ Markowitz ${ }^{4}$, Philip Hugenholtz ${ }^{1,8}$, Nikos C. Kyrpides ${ }^{1}$, Hans-Peter Klenk ${ }^{2}$, and Alla Lapidus ${ }^{1 *}$ \\ ${ }^{1}$ DOE Joint Genome Institute, Walnut Creek, California, USA \\ ${ }^{2}$ DSMZ - German Collection of Microorganisms and Cell Cultures GmbH, Braunschweig, \\ Germany \\ ${ }^{3}$ Los Alamos National Laboratory, Bioscience Division, Los Alamos, New Mexico, USA \\ ${ }^{4}$ Biological Data Management and Technology Center, Lawrence Berkeley National \\ Laboratory, Berkeley, California, USA \\ ${ }^{5}$ Oak Ridge National Laboratory, Oak Ridge, Tennessee, USA \\ ${ }^{6} \mathrm{HZI}$ - Helmholtz Centre for Infection Research, Braunschweig, Germany \\ ${ }^{7}$ University of California Davis Genome Center, Davis, California, USA \\ ${ }^{8}$ Australian Centre for Ecogenomics, School of Chemistry and Molecular Biosciences, The \\ University of Queensland, Brisbane, Australia \\ *Corresponding author: Alla Lapidus
}

Keywords: aerobic, chemoorganotrophic, non-motile, thermophilic, facultatively halophilic, alkaliphilic, radiation resistant, Gram-indeterminate, spherical-shaped, Trueperaceae, GEBA

Truepera radiovictrix Albuquerque et al. 2005 is the type species of the genus Truepera within the phylum "Deinococcus/Thermus". T. radiovictrix is of special interest not only because of its isolated phylogenetic location in the order Deinococcales, but also because of its ability to grow under multiple extreme conditions in alkaline, moderately saline, and high temperature habitats. Of particular interest is the fact that, $T$. radiovictrix is also remarkably resistant to ionizing radiation, a feature it shares with members of the genus Deinococcus. This is the first completed genome sequence of a member of the family Trueperaceae and the fourth type strain genome sequence from a member of the order Deinococcales. The 3,260,398 bp long genome with its 2,994 protein-coding and 52 RNA genes consists of one circular chromosome and is a part of the Genomic Encyclopedia of Bacteria and Archaea project.

\section{Introduction}

Strain RQ-24T (= DSM 17093 = LMG 22925 = CIP 108686) is the type strain of Truepera radiovictrix which is the sole and type species of the genus Truepera $[1,2]$. The generic name of strain RQ-24T derives from the name "Trüper", in honor of the German microbiologist Hans G. Trüper. The species epithet is derived from the Latin radiovictrix, the vanquisher of radiation [1]. Strain RQ-24T was isolated in 2005 from a hot spring within a geothermal area located along an almost vertical wall and dry bed of the stream Ribeira Quente, about $500 \mathrm{~m}$ south-east of a geothermal area on the eastern edge of the town Furnas, Azores [1]. Close to the area where RQ-24T was isolated an accompanying isolate, strain TU-8, was also obtained [1]. Strains RQ-24 ${ }^{\mathrm{T}}$ and TU-8 share most physiological features as well as identical 16S rRNA sequence. The two T. radiovictrix strains also share many chemotaxonomic and physiological characteristics with the members of the genus Deinococcus, including the extreme resistance to ionizing radiation. Here we present a summary classification and a set of features for T. radiovictrix RQ-24T, together with the description of the complete genomic sequencing and annotation. 


\section{Classification and features}

Figure 1 shows the phylogenetic neighborhood of strain RQ-24T in a $16 \mathrm{~S}$ rRNA based tree. The sequences of the two 16S rRNA gene copies in the genome differ from each other by four nucleotides, and differ by up to three nucleotides from the previously published $16 \mathrm{~S}$ rRNA sequence (DQ022076), which contains one ambiguous base call.

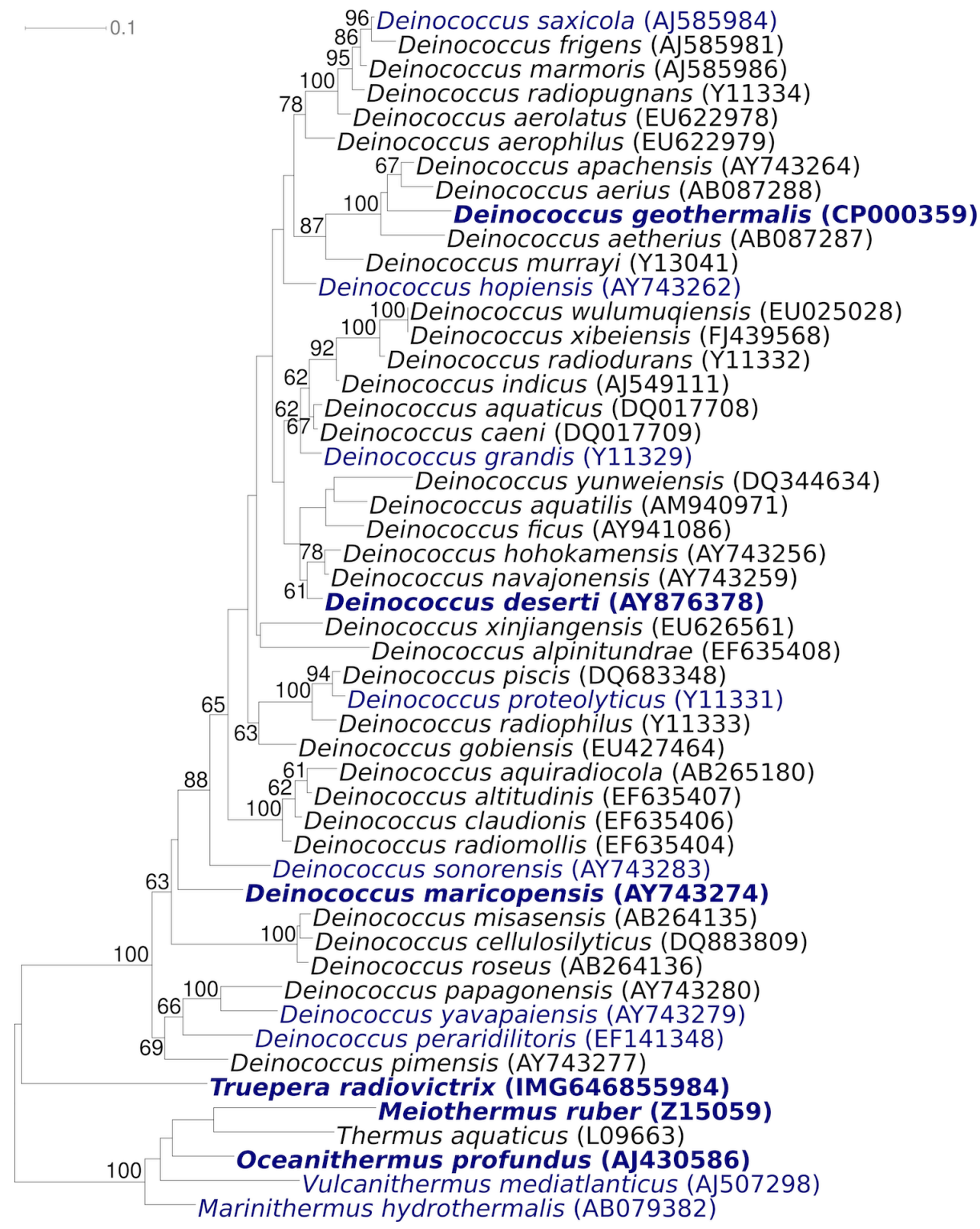

Figure 1. Phylogenetic tree highlighting the position of $T$. radiovictrix relative to the type strains of the other species within the class 'Deinococci'. The tree was inferred from 1,457 aligned characters $[3,4]$ of the $16 \mathrm{~S}$ rRNA gene sequence under the maximum likelihood criterion [5] and rooted in accordance with the current taxonomy. The branches are scaled in terms of the expected number of substitutions per site. Numbers above branches are support values from 1,000 bootstrap replicates [6] if larger than $60 \%$. Lineages with type strain genome sequencing projects registered in GOLD [7] are shown in blue, publicly available complete genome sequences [CP002361, Oceanithermus profundus] and published genomes [8-11] in bold. The genome of $D$. radidurans published by White at al. in 1999 [12] later turned out to be not from the type strain [13]. 
A representative genomic $16 \mathrm{~S}$ rRNA sequence of strain RQ-2 $2^{\mathrm{T}}$ was compared using NCBI BLAST under default settings (e.g., considering only the high-scoring segment pairs (HSPs) from the best 250 hits) with the most recent release of the Greengenes database [14] and the relative frequencies, weighted by BLAST scores, of taxa and keywords (reduced to their stem [15]) were determined. The five most frequent genera were Deinococcus (84.0\%), Truepera (8.0\%), Oceanithermus (6.3\%), Thiocapsa (0.9\%) and Thiobaca $(0.8 \%)$ (98 hits in total). Regarding the two hits to sequences from members of the species, the average identity within HSPs was $99.7 \%$, whereas the average coverage by HSPs was $97.6 \%$. Among all other species, the one yielding the highest score was $O$. profundus, which corresponded to an identity of $87.5 \%$ and an HSP coverage of $61.9 \%$. The highest-scoring environmental sequence was EU924247 ('Microbiology and geochemistry Little Hot Creek hot spring sediment temperature 80 degrees C clone LHC1 L4 D07'), which showed an identity of $96.6 \%$ and an HSP coverage of $91.4 \%$. The five most frequent keywords within the labels of environmental samples which yielded hits were 'rock' (3.1\%), 'microbi' (2.9\%), 'skin' (2.1\%), 'soil' $(1.9 \%)$ and 'air' (1.6\%) (152 hits in total). The five most frequent keywords within the labels of environmental samples which yielded hits of a higher score than the highest scoring species were 'rock' (4.2\%), 'microbi' (2.7\%), 'air' (2.4\%), 'soil' (2.1\%) and 'cabin/commerci' (2.0\%) (60 hits in total), indicating the existence of close relatives of the strain also in less extreme habitats.

The non-motile, red-pigmented cells of RQ-24T are spherical and approximately $1.25-2.0 \mu \mathrm{m}$ in diameter, forming predominantly pairs or tetrads (Figure 2 and Table 1). Ultrathin sections of the cytoplasm revealed ribosomes, a fibrillar nucleoid and tubular structures of unknown nature, as well as three distinct layers of the cell wall, the innermost layer being thin, the outermost layer being as thick as $20-90 \mathrm{~nm}$, both being electron dense [1]. Strain RQ-24 grows best at around $50^{\circ} \mathrm{C}$, but not at $20^{\circ} \mathrm{C}$ or $60^{\circ} \mathrm{C}$, with $1.0 \% \mathrm{NaCl}$ in Thermus medium or Degryse medium 162. The acceptable salinity range supporting growth is up to $6.0 \%$ $\mathrm{NaCl}$. Optimum $\mathrm{pH}$ is 7.5-9.5, with growth detected up to $\mathrm{pH} 11.2$, but no growth was detected at $\mathrm{pH} 6.0$ [1]. Strains RQ-24 ${ }^{\mathrm{T}}$ and TU-8 are the most alkaliphilic members of the phylum "Deinococcus/Thermus" [1]. Both strains are oxidase and catalase positive and use a wide range of carbohydrates, organic acids or amino acids as carbon and energy sources with a respiratory metabolism (Table 1). Unexpectedly, strains RQ-24T and TU-8 are capable of fermenting glucose to lactate via homolactic fermentation [1]. Also, both strains are extremely resistant to gamma irradiation, with $60 \%$ survival rate after exposure to $5.0 \mathrm{kGy}$ [1]. They share this radiation resistance trait with strains from the genus Deinococcus, Kineococcus radiotolerans and the actinobacterial genus $R u$ brobacter [25].

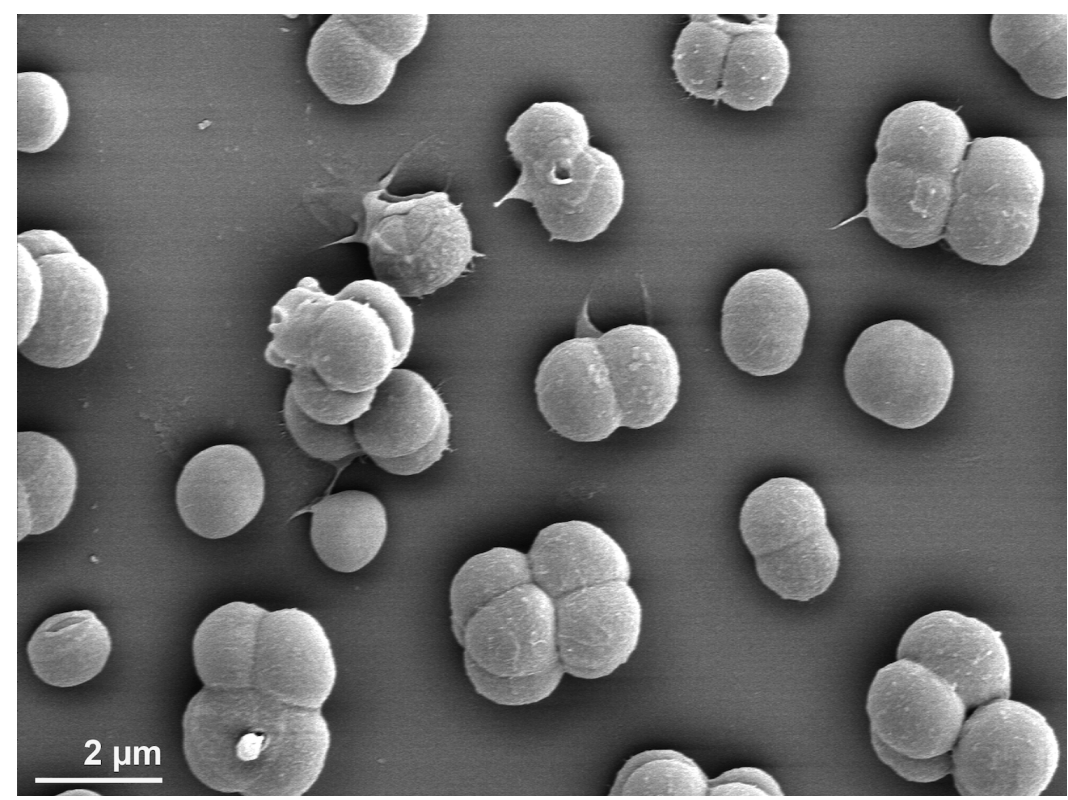

Figure 2. Scanning electron micrograph of T. radiovictrix RQ-24 ${ }^{\top}$ 
Table 1. Classification and general features of T. radiovictrix RQ-24 ${ }^{\top}$ according to the MIGS recommendations [16].

\begin{tabular}{|c|c|c|c|}
\hline MIGS ID & Property & Term & Evidence code \\
\hline & \multirow{8}{*}{ Current classification } & Domain Bacteria & TAS [17] \\
\hline & & Phylum "Deinococcus-Thermus" & TAS $[18,19]$ \\
\hline & & Class Deinococci & TAS $[20,21]$ \\
\hline & & Order Deinococcales & TAS [22] \\
\hline & & Family Trueperaceae & TAS $[1,2]$ \\
\hline & & Genus Truepera & TAS $[1,2]$ \\
\hline & & Species Truepera radiovictrix & TAS $[1,2]$ \\
\hline & & Type strain RQ-24 & TAS [1] \\
\hline & Gram stain & indeterminate & TAS [1] \\
\hline & Cell shape & spherical, mostly in pairs or tetrads & TAS [1] \\
\hline & Motility & non-motile & TAS [1] \\
\hline & Sporulation & none & TAS [1] \\
\hline & Temperature range & $25-55^{\circ} \mathrm{C}$ & TAS [1] \\
\hline & Optimum temperature & $50^{\circ} \mathrm{C}$ & TAS [1] \\
\hline & Salinity & $1 \% \mathrm{NaCl}$ & TAS [1] \\
\hline \multirow[t]{3}{*}{ MIGS-22 } & Oxygen requirement & strictly aerobic & TAS [1] \\
\hline & Carbon source & carbohydrates, organic acids, amino acids & TAS [1] \\
\hline & Energy source & chemoorganotrophic & TAS [1] \\
\hline MIGS-6 & Habitat & hot spring & TAS [1] \\
\hline MIGS-15 & Biotic relationship & free-living & TAS [1] \\
\hline \multirow[t]{3}{*}{ MIGS-14 } & Pathogenicity & none & NAS \\
\hline & Biosafety level & 1 & TAS [23] \\
\hline & Isolation & hot spring runoff in geothermal area & TAS [1] \\
\hline MIGS-4 & Geographic location & $\begin{array}{l}\text { River Ribeira Quente, near Furnas, } \\
\text { Island of Sao Miguel, Azores }\end{array}$ & TAS [1] \\
\hline MIGS-5 & Sample collection time & 2004 or before & TAS [1] \\
\hline MIGS-4.1 & Latitude & 37.95 & NAS \\
\hline MIGS-4.2 & Longitude & -25.49 & NAS \\
\hline MIGS-4.3 & Depth & not reported & \\
\hline MIGS-4.4 & Altitude & 109 meter & NAS \\
\hline
\end{tabular}

Evidence codes - IDA: Inferred from Direct Assay (first time in publication); TAS: Traceable Author Statement (i.e., a direct report exists in the literature); NAS: Non-traceable Author Statement (i.e., not directly observed for the living, isolated sample, but based on a generally accepted property for the species, or anecdotal evidence). These evidence codes are from of the Gene Ontology project [24]. If the evidence code is IDA, then the property was directly observed by one of the authors or an expert mentioned in the acknowledgements.

\section{Chemotaxonomy}

All attempts to identify a peptidoglycan of strain RQ-24T failed [1]. The polar lipids comprised as complex mixture of glycolipids and phospholipids, although no attempt has been made to compare them with the characteristic compounds found in members of the orders Deinococcales or Thermales. The major respiratory quinone is menaquinone 8 (MK-8). The fatty acids are predominantly saturated branched acids of which anteiso- $\mathrm{C}_{15: 0}$ (38.6\%), anteiso- $\mathrm{C}_{17}(17.2 \%)$ and iso- $\mathrm{C}_{17: 0}(16.6 \%)$ as well as iso- $\mathrm{C}_{16: 0}(6.9 \%)$. One acyl compound has an equivalent chain length (ECL) consistent with iso- $\mathrm{C}_{18: 0}$ 1,2-diol and another compound with ECL 16.090 probably representing iso- $\mathrm{C}_{15: 0}$ diol. The presence of the long-chain 1,2 diols is unknown in members of the genus Deinococcus (although the methods normally used would not identify them), while they are found in some other members of the genera Thermus and Meiothermus. Despite the fact that members of the species $T$. radiovictrix is described as being red pigmented there is no data on the nature of the pigments. 


\section{Genome sequencing and annotation Genome project history}

This organism was selected for sequencing on the basis of its phylogenetic position [26], and is part of the Genomic Encyclopedia of Bacteria and Archaea project [27]. The genome project is deposited in the Genomes OnLine Database [7] and the complete genome sequence is deposited in GenBank. Sequencing, finishing and annotation were performed by the DOE Joint Genome Institute (JGI). A summary of the project information is shown in Table 2.

Table 2. Genome sequencing project information

\begin{tabular}{|c|c|c|}
\hline MIGS ID & Property & Term \\
\hline MIGS-31 & Finishing quality & Finished \\
\hline MIGS-28 & Libraries used & $\begin{array}{l}\text { Three genomic libraries: one } 454 \text { pyrosequence stan- } \\
\text { dard library, one } 454 \text { PE library (18 kb insert size), } \\
\text { one Illumina library }\end{array}$ \\
\hline MIGS-29 & Sequencing platforms & Illumina GAii, 454 GS FLX Titanium \\
\hline MIGS-31.2 & Sequencing coverage & $70.6 \times$ Illumina; $82.5 \times$ pyrosequence \\
\hline MIGS-30 & Assemblers & $\begin{array}{l}\text { Newbler version 2.1-PreRelease-4-28-2009-gcc- } \\
\text { 3.4.6-threads, Velvet, phrap }\end{array}$ \\
\hline \multirow[t]{6}{*}{ MIGS-32 } & Gene calling method & Prodigal 1.4, GenePRIMP \\
\hline & INSDC ID & СР002049 \\
\hline & Genbank Date of Release & May 28, 2010 \\
\hline & GOLD ID & Gc01303 \\
\hline & NCBI project & 38371 \\
\hline & Database: IMG-GEBA & 2502957036 \\
\hline \multirow[t]{2}{*}{ MIGS-13 } & Source material identifier & DSM 17093 \\
\hline & Project relevance & Tree of Life, GEBA \\
\hline
\end{tabular}

\section{Growth conditions and DNA isolation}

T. radiovictrix RQ-24T, DSM 17093, was grown in DSMZ medium 1033 (Thermus Medium) [28] at $50^{\circ} \mathrm{C}$. DNA was isolated from $0.5-1 \mathrm{~g}$ of cell paste using MasterPure Gram-positive DNA purification kit (Epicentre MGP04100) following the standard protocol as recommended by the manufacturer, with modification st/LALM for cell lysis as described in $\mathrm{Wu}$ et al. [27]. DNA of strain RQ-24T is available through the DNA Bank Network $[29,30]$.

\section{Genome sequencing and assembly}

The genome was sequenced using a combination of Illumina and 454 sequencing platforms. All general aspects of library construction and sequencing can be found at the JGI website [31]. Pyrosequencing reads were assembled using the Newbler assembler version 2.1-PreRelease-4-282009-gcc-3.4.6-threads (Roche). The initial Newbler assembly consisting of 75 contigs in five scaffolds was converted into a phrap [32] assembly by making fake reads from the consensus, to collect the read pairs in the 454 paired end library. Illumina GAii sequencing data (230.2 Mb) was assembled with Velvet [33] and the consensus se- quences were shredded into $1.5 \mathrm{~kb}$ overlapped fake reads and assembled together with the 454 data. The 454 draft assembly was based on 268.9 $\mathrm{Mb} 454$ draft data and all of the 454 paired end data. Newbler parameters are -consed -a 50 -l 350 -g -m -ml 20. The Phred/Phrap/Consed software package [32] was used for sequence assembly and quality assessment in the subsequent finishing process. After the shotgun stage, reads were assembled with parallel phrap (High Performance Software, LLC). Possible mis-assemblies were corrected with gapResolution [31], Dupfinisher, or sequencing cloned bridging PCR fragments with subcloning or transposon bombing (Epicentre Biotechnologies, Madison, WI) [34]. Gaps between contigs were closed by editing in Consed, by PCR and by Bubble PCR primer walks (J.-F.Chang, unpublished). A total of 336 additional reactions were necessary to close gaps and to raise the quality of the finished sequence. Illumina reads were also used to correct potential base errors and increase consensus quality using a software Polisher developed at JGI [35]. The error rate of the completed genome sequence is less than 1 in 100,000. 
Together, the combination of the Illumina and 454 sequencing platforms provided $153.1 \times$ coverage of the genome. The final assembly contained 736,380 pyrosequence and 6,393,275 Illumina reads.

\section{Genome annotation}

Genes were identified using Prodigal [36] as part of the Oak Ridge National Laboratory genome annotation pipeline, followed by a round of manual curation using the JGI GenePRIMP pipeline [37]. The predicted CDSs were translated and used to search the National Center for Biotechnology Information (NCBI) nonredundant database, UniProt, TIGR-Fam, Pfam, PRIAM, KEGG, COG, and InterPro databases. Additional gene prediction anal- ysis and functional annotation was performed within the Integrated Microbial Genomes - Expert Review (IMG-ER) platform [38].

\section{Genome properties}

The genome consists of a 3,260,398 bp long chromosome with a $\mathrm{G}+\mathrm{C}$ content of $68.1 \%$ (Figure 3 and Table 3). Of the 3,046 genes predicted, 2,994 were protein-coding genes, and 52 RNAs; 49 pseudogenes were also identified. The majority of the protein-coding genes (73.4\%) were assigned with a putative function while the remaining ones were annotated as hypothetical proteins. The distribution of genes into COGs functional categories is presented in Table 4.

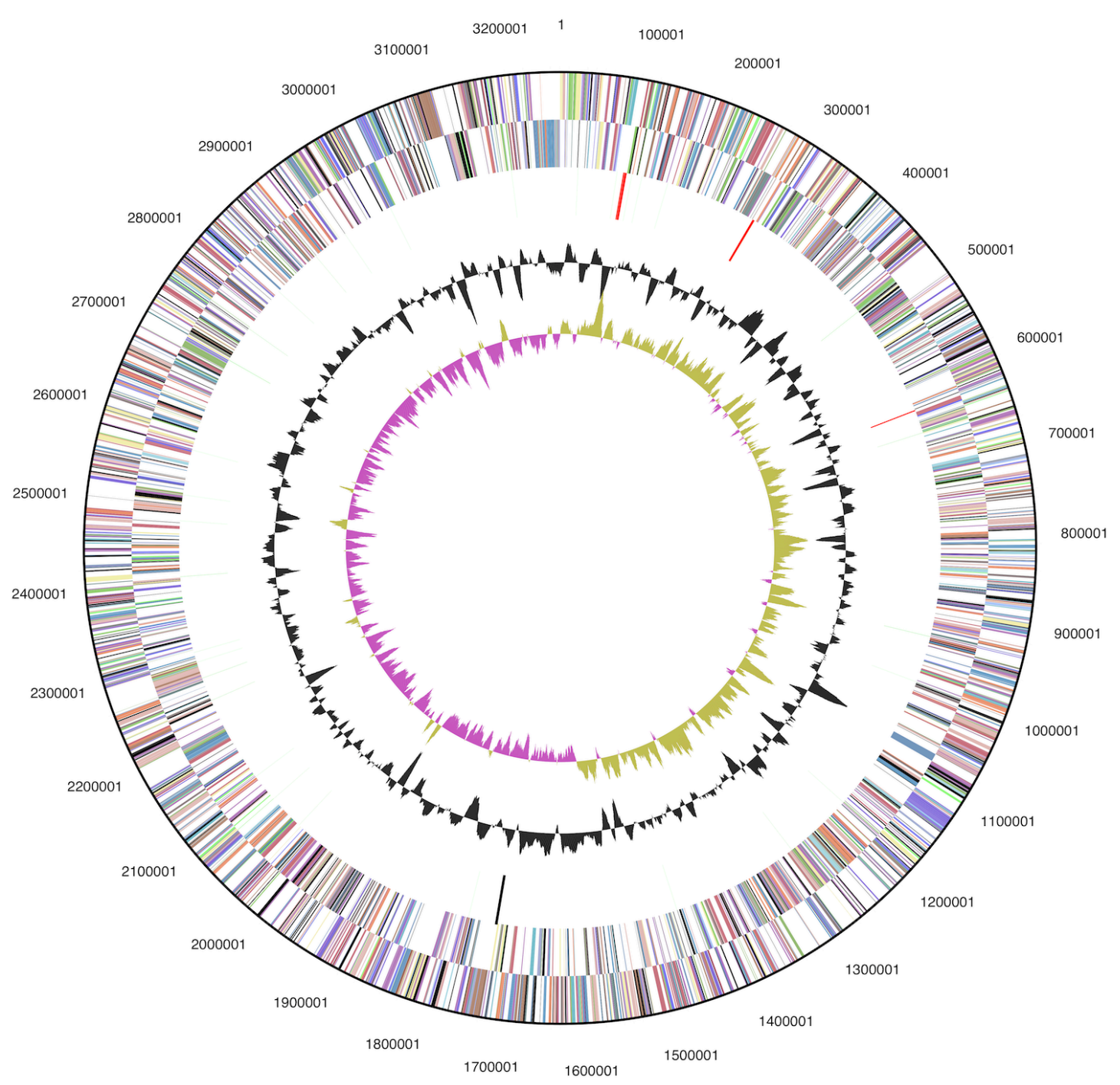

Figure 3. Graphical circular map of the chromosome. From outside to the center: Genes on forward strand (color by COG categories), Genes on reverse strand (color by COG categories), RNA genes (tRNAs green, rRNAs red, other RNAs black), GC content, GC skew. 
Table 3. Genome Statistics

\begin{tabular}{lrr}
\hline Attribute & Value & \% of Total \\
\hline Genome size (bp) & $3,260,398$ & $100.00 \%$ \\
DNA coding region (bp) & $2,862,171$ & $87.79 \%$ \\
DNA G+C content (bp) & $2,221,603$ & $68.14 \%$ \\
Number of replicons & 1 & \\
Extrachromosomal elements & 0 & \\
Total genes & 3,046 & $100.00 \%$ \\
RNA genes & 52 & $1.71 \%$ \\
rRNA operons & 2 & \\
Protein-coding genes & 2,994 & $98.29 \%$ \\
Pseudo genes & 49 & $1.61 \%$ \\
Genes with function prediction & 2,235 & $73.37 \%$ \\
Genes in paralog clusters & 370 & $12.15 \%$ \\
Genes assigned to COGs & 2,272 & $74.59 \%$ \\
Genes assigned Pfam domains & 2,385 & $78.30 \%$ \\
Genes with signal peptides & 1,177 & $38.64 \%$ \\
Genes with transmembrane helices & 709 & $23.28 \%$ \\
CRISPR repeats & 9 & \\
\hline
\end{tabular}

Table 4. Number of genes associated with the general COG functional categories

\begin{tabular}{lrrl}
\hline Code & value & \%age & Description \\
\hline J & 149 & 5.9 & Translation, ribosomal structure and biogenesis \\
A & 0 & 0.0 & RNA processing and modification \\
K & 126 & 5.0 & Transcription \\
L & 135 & 5.4 & Replication, recombination and repair \\
B & 2 & 0.1 & Chromatin structure and dynamics \\
D & 29 & 1.2 & Cell cycle control, cell division, chromosome partitioning \\
Y & 0 & 0.0 & Nuclear structure \\
V & 40 & 1.6 & Defense mechanisms \\
T & 93 & 3.7 & Signal transduction mechanisms \\
M & 128 & 5.1 & Cell wall/membrane/envelope biogenesis \\
N & 14 & 0.6 & Cell motility \\
Z & 0 & 0.0 & Cytoskeleton \\
W & 0 & 0.0 & Extracellular structures \\
U & 34 & 1.4 & Intracellular trafficking, secretion, and vesicular transport \\
O & 82 & 3.3 & Posttranslational modification, protein turnover, chaperones \\
C & 148 & 5.9 & Energy production and conversion \\
G & 244 & 9.7 & Carbohydrate transport and metabolism \\
E & 271 & 10.7 & Amino acid transport and metabolism \\
F & 79 & 3.1 & Nucleotide transport and metabolism \\
H & 104 & 4.1 & Coenzyme transport and metabolism \\
I & 84 & 3.3 & Lipid transport and metabolism \\
P & 170 & 6.7 & Inorganic ion transport and metabolism \\
Q & 63 & 2.5 & Secondary metabolites biosynthesis, transport and catabolism \\
R & 343 & 13.6 & General function prediction only \\
S & 187 & 7.4 & Function unknown \\
- & 774 & 25.4 & Not in CoGs \\
\hline
\end{tabular}




\section{Acknowledgements}

We would like to gratefully acknowledge the help of Helga Pomrenke (DSMZ) for growing T. radiovictrix cultures. This work was performed under the auspices of the US Department of Energy Office of Science, Biological and Environmental Research Program, and by the University of California, Lawrence Berkeley National Laboratory under contract No. DE-AC02-05CH11231,

\section{References}

1. Albuquerque L, Simoes C, Nobre MF, Pino NM, Battista JR, Silva MT, Rainey FA, Da Costa MS. Truepera radiovictrix gen. nov., sp. nov., a new radiation resistant species and the proposal of Trueperaceae fam. nov. FEMS Microbiol Lett 2005;

247:161-169. PubMed doi:10.1016/j.femsle.2005.05.002

2. Euzéby J. Validation of publication of new names and new combinations previously effectively published outside the IJSEM. Int / Syst Evol Microbiol 2005; 55:1743-1745. PubMed doi:10.1099/ijs.0.63996-0

3. Castresana J. Selection of conserved blocks from multiple alignments for their use in phylogenetic analysis. Mol Biol Evol 2000; 17:540-552. PubMed

4. Lee C, Grasso C, Sharlow MF. Multiple sequence alignment using partial order graphs. Bioinformatics 2002; 18:452-464. PubMed doi:10.1093/bioinformatics/18.3.452

5. Stamatakis A, Hoover P, Rougemont J. A rapid bootstrap algorithm for the RAxML Web servers. Syst Biol 2008; 57:758-771. PubMed doi:10.1080/10635150802429642

6. Pattengale ND, Alipour M, Bininda-Emonds ORP, Moret BME, Stamatakis A. How many bootstrap replicates are necessary? Lect Notes Comput Sci 2009; 5541:184-200. doi:10.1007/978-3-642$\underline{02008-7 \quad 13}$

7. Liolios K, Chen IM, Mavromatis K, Tavernarakis N, Hugenholtz P, Markowitz VM, Kyrpides NC. The Genomes On Line Database (GOLD) in 2009: status of genomic and metagenomic projects and their associated metadata. Nucleic Acids Res 2010; 38:D346-D354. PubMed doi:10.1093/nar/gkp848

8. de Groot A, Dulermo R, Ortet P, Blanchard L, Guerin P, Fernandez B, Vacherie B, Dossat C, Jolivet $\mathrm{E}$, Siguier $\mathrm{P}$, et al. Alliance of proteomics and genomics to unravel the specificities of Sahara bacterium Deinococcus deserti. PLoS Genet 2009; 5:e1000434. PubMed doi:10.1371/journal.pgen.1000434
Lawrence Livermore National Laboratory under Contract No. DE-AC52-07NA27344, and Los Alamos National Laboratory under contract No. DE-AC0206NA25396, UT-Battelle and Oak Ridge National Laboratory under contract DE-AC05-000R22725, as well as German Research Foundation (DFG) INST 599/1-2.

9. Makarova KS, Omelchenko MV, Gaidamakova EK, Matrosova VY, Vasilenko A, Zhai M, Lapidus A, Copeland A, Kim E, Land M, et al. Deinococcus geothermalis: the pool of extreme radiation resistance genes shrinks. PLOS ONE 2007; 2:e955. PubMed doi:10.1371/journal.pone.0000955

10. Pukall R, Zeytun A, Lucas A, Lapidus A, Hammon N, Deshpande S, Nolan M, Cheng JF, Pitluck S, Liolios K, et al. Complete genome sequence of Deinococcus maricopensis type strain $\left(\mathrm{LB}-34^{\top}\right)$. Stand Genomic Sci 2011; (next issue).

11. Tindall BJ, Sikorski J, Lucas S, Goltsman E, Copeland A, Glavina Del Rio T, Nolan M, Tice H, Cheng $\mathrm{JF}$, Han C, et al. Complete genome sequence of Meiothermus ruber type strain $\left(21^{\top}\right)$. Stand Genomic Sci 2010; 3:26-36. PubMed doi:10.4056/sigs.1032748

12. White O, Eisen JA, Heidelberg JF, Hickey EK, Peterson JD, Dodson RJ, Haft DH, Gwinn ML, Nelson WC, Richardson DL, et al. Genome sequence of the radioresistant bacterium Deinococcus radiodurans R1. Science 1999; 286:1571-1577. PubMed doi:10.1126/science.286.5444.1571

13. White $\mathrm{O}$, Eisen JA, Heidelberg JF, Hickey EK, Peterson JD, Dodson RJ, Haft DH, Gwinn ML, Nelson WC, Richardson DL, et al. Erratum: Genome sequence of the radioresistant bacterium Deinococcus radiodurans R1. Science 2004; 303:15711577.

14. DeSantis TZ, Hugenholtz $P$, Larsen $N$, Rojas $M$, Brodie EL, Keller K, Huber T, Dalevi D, Hu P, Andersen GL. Greengenes, a Chimera-checked 16S rRNA Gene Database and Workbench Compatible with ARB. Appl Environ Microbiol 2006; 72:50695072. PubMed doi:10.1128/AEM.03006-05

15. Porter MF. An algorithm for suffix stripping. Program: electronic library and information systems 1980; 14:130-137. doi:10.1108/eb046814

16. Field D, Garrity G, Gray T, Morrison N, Selengut J, Sterk P, Tatusova T, Thomson N, Allen MJ, Angiuoli SV, et al. The minimum information about a genome sequence (MIGS) specification. Nat Biotech- 
nol 2008; 26:541-547. PubMed

$\underline{\text { doi: } 10.1038 / \mathrm{nbt} 1360}$

17. Woese CR, Kandler O, Wheelis ML. Towards a natural system of organisms: proposal for the domains Archaea, Bacteria, and Eucarya. Proc Natl Acad Sci USA 1990; 87:4576-4579. PubMed doi:10.1073/pnas.87.12.4576

18. Weisburg WG, Giovannoni SJ, Woese CR. The Deinococcus-Thermus phylum and the effect of rRNA composition on phylogenetic tree construction. Syst Appl Microbiol 1989; 11:128-134. PubMed

19. Garrity GM, Holt JG. 2001. Taxonomic outline of the Archaea and Bacteria, p. 155-166. In G. M. Garrity, D. R. Boone, and R. W. Castenholz (ed.), Bergey's Manual of Systematic Bacteriology, 2nd ed, vol. 1. Springer, New York.

20. List Editor. Validation List no. 85. Validation of publication of new names and new combinations previously effectively published outside the IJSEM. Int J Syst Evol Microbiol 2002; 52:685-690. PubMed doi:10.1099/ijs.0.02358-0

21. Garrity GM, Holt JG. Class I. Deinococci class. nov. In: Garrity GM, Boone DR, Castenholz RW (eds), Bergey's Manual of Systematic Bacteriology, Second Edition, Volume 1, Springer, New York, 2001, p. 395.

22. Rainey FA, Nobre MF, Schumann P, Stackebrandt E, da Costa MS. Phylogenetic diversity of the deinococci as determined by $16 \mathrm{~S}$ ribosomal DNA sequence comparison. Int I Syst Bacteriol 1997; 47:510-514. PubMed doi:10.1099/00207713-472-510

23. Classification of bacteria and archaea in risk groups. http://www.baua.de TRBA 466.

24. Ashburner M, Ball CA, Blake JA, Botstein D, Butler $\mathrm{H}$, Cherry JM, Davis AP, Dolinski K, Dwight SS, Eppig JT, et al. Gene Ontology: tool for the unification of biology. Nat Genet 2000; 25:25-29. PubMed doi:10.1038/75556

25. Ferreira AC, Nobre MF, Moore E, Rainey FA, Battista JR, da Costa MS. Characterization and radiation resistance of new isolates of Rubrobacter radiotolerans and Rubrobacter xylanophilus. Extremophiles 1999; 3:235-238. PubMed doi:10.1007/s007920050121

26. Klenk HP, Göker M. En route to a genome-based classification of Archaea and Bacteria? Syst Appl Microbiol 2010; 33:175-182. PubMed doi:10.1016/j.syapm.2010.03.003
27. Wu D, Hugenholtz P, Mavromatis K, Pukall R, Dalin E, Ivanova NN, Kunin V, Goodwin L, Wu M, Tindall BJ, et al. A phylogeny-driven genomic encyclopaedia of Bacteria and Archaea. Nature 2009; 462:1056-1060. PubMed doi:10.1038/nature08656

28. List of growth media used at DSMZ: http://www.dsmz.de/microorganisms/media_list.ph p.

29. Gemeinholzer B, Dröge G, Zetzsche H, Haszprunar G, Klenk HP, Güntsch A, Berendsohn WG, Wägele JW. The DNA Bank Network: the start from a German initiative. Biopreservation and Biobanking (In press).

30. DNA bank Network. http://www.dnabanknetwork.org

31. The DOE Joint Genome Institute. http://www.jgi.doe.gov

32. Phrap and Phred for Windows, MacOS, Linux, and Unix. http://www.phrap.com

33. Zerbino DR, Birney E. Velvet: algorithms for de novo short read assembly using de Bruijn graphs. Genome Res 2008; 18:821-829. PubMed doi:10.1101/gr.074492.107

34. Sims D, Brettin T, Detter JC, Han C, Lapidus A, Copeland A, Glavina Del Rio T, Nolan M, Chen F, Lucas $\mathrm{S}$, et al. Complete genome sequence of $\mathrm{Ky}$ tococcus sedentarius type strain $\left(541^{\top}\right)$. Stand Genomic Sci 2009; 1:12-20. PubMed doi:10.4056/sigs.761

35. Lapidus A, LaButti K, Foster B, Lowry S, Trong S, Goltsman E. POLISHER: An effective tool for using ultra short reads in microbial genome assembly and finishing. AGBT, Marco Island, FL, 2008.

36. Hyatt D, Chen GL, LoCascio PF, Land ML, Larimer FW, Hauser LJ. Prodigal: prokaryotic gene recognition and translation initiation site identification. BMC Bioinformatics 2010; 11:119. PubMed doi:10.1186/1471-2105-11-119

37. Pati A, Ivanova NN, Mikhailova N, Ovchinnikova G, Hooper SD, Lykidis A, Kyrpides NC. GenePRIMP: a gene prediction improvement pipeline for prokaryotic genomes. Nat Methods 2010; 7:455-457. PubMed doi:10.1038/nmeth.1457

38. Markowitz VM, Ivanova NN, Chen IMA, Chu K, Kyrpides NC. IMG ER: a system for microbial genome annotation expert review and curation. Bioinformatics 2009; 25:2271-2278. PubMed doi:10.1093/bioinformatics/btp393 